\title{
Specimen records for North American Lepidoptera (Insecta) in the Oregon State Arthropod Collection. Cossoidea: Cossidae Leach, 1815 and Sesiidae Boisduval, 1828
}

Jon H. Shepard

Paul C. Hammond

Christopher J. Marshall

Oregon State Arthropod Collection, Department of Integrative Biology, Oregon State University, Corvallis OR 97331

Cite this work, including the attached dataset, as:

Shepard, J. H, P. C. Hammond, C. J. Marshall. 2019. Specimen records for North American Lepidoptera (Insecta) in the Oregon State Arthropod Collection. Cossoidea: Cossidae Leach, 1815 and Sesiidae Boisduval, 1828. Catalog: Oregon State Arthropod Collection 3(2) (beta version) http:/ / dx.doi.org/10.5399/osu/cat osac.3.2.4592

\section{Introduction}

These records were generated using funds from the LepNet project (Seltmann et. al., 2017) - a national effort to create digital records for North American Lepidoptera. The dataset published herein contains the label data for all North American specimens of Cossidae and Sesiidae residing at the Oregon State Arthropod Collection as of March 2019. A beta version of these data records will be made available on the OSAC server (http:/ / osac.oregonstate.edu/IPT) at the time of this publication. The beta version will be replaced in the near future with an official release (version 1.0), which will be archived as a supplemental file to this paper.

\section{Methods}

Basic digitization protocols and metadata standards can be found in (Shepard et al. 2018). Much of the newer material was identified by two specilaists on Sesiidae: Mr. John Vernon and Mr. Benjamin G. Thompson. Identifications were reviewed by Jon Shepard and Paul Hammond using Barnes and McDunnough (1911) and the Digital Guide to Moth Identification (Moths Protographers Group, 2019). Nomenclature follows that of Hodges (1983) and the Moth Photographers Group (2019) and was checked again using the species matching tool produced by GBIF (https:/ / www.gbif.org/tools/ species-lookup).

\section{Results}

As of March 2019, the collection possessed 181 specimens of North American Cossidae, belonging to 8 known species classified into 6 genera: Comadia Barnes \& McDunnough 1911, Cossus Fabricius 1793, Givira Walker 1856, Prionoxystus Grote 1882, Toronia Barnes \& McDunnough 1911, and Zeuzera Latreille 1804 (Table 1)

The collection's holdings for Sesiidae are more extensive, including 1954 specimens, belonging to 82 distinct species, classified among 17 genera: Albuna Edwards 1881, Alcathoe Edwards, 1882, Carmenta Edwards 1881, Euhagena Edwards 1881, Hymenoclea Engelhardt, 1946, Melittia Hübner 1819, Palmia Beutenmüller, 1896, Paranthrene Strand, 1916, Pennisetia Dehne, 1850, Penstemonia Engelhardt, 
1946, Podosesia Möschler, 1879, Sannina Walker, 1856, Sesia Fabricius 1775, Sophona Walker, 1856, Synanthedon Hübner, 1819, Vitacea Engelhardt, 1946, Zenodoxus Grote \& Robinson, 1868 (Table 2).

Table 1. Species inventory of OSAC North American Cossidae

Taxon
\begin{tabular}{|l|c|}
\hline Acossus centerensis Lintner, 1877 & 4 \\
\hline Acossus populi (Walker, 1856) & 65 \\
\hline Comadia bertholdi Grote, 1880 & 13 \\
\hline Comadia henrici Grote, 1882 & 1 \\
\hline Comadia undetermined & 9 \\
\hline Givira marga Barnes \& McDunnough, 1910 & 2 \\
\hline Givira undetermined & 12 \\
\hline Prionoxystus robiniae Peck, 1818 & 69 \\
\hline Toronia perplexa (Neumogen \& Dyar, 1893) & 5 \\
\hline Zeuzera pyrina (Linnaeus, 1761) & 1 \\
\hline \multicolumn{2}{|c|}{ total } \\
\hline
\end{tabular}

Table 2. Species inventory of OSAC North American Sesiidae

\begin{tabular}{|c|c|}
\hline Taxon & \# specimens \\
\hline Albuna fraxini Edwards, 1881 & 14 \\
\hline Albuna pyramidalis Walker, 1856 & 43 \\
\hline Alcathoe autumnalis Engelhardt, 1946 & 2 \\
\hline Alcathoe carolinensis Engelhardt, 1925 & 14 \\
\hline Alcathoe caudata Harris, 1839 & 11 \\
\hline Alcathoe pepsioides Engelhardt, 1925 & 2 \\
\hline Alcathoe verrugo (Druce, 1884) & 1 \\
\hline Carmenta bassiformis Walker, 1856 & 11 \\
\hline Carmenta corni Edwards, 1881 & 11 \\
\hline Carmenta engelhardti Duckworth \& Eichlin, 1973 & 3 \\
\hline Carmenta giliae Edwards, 1881 & 1 \\
\hline Carmenta ithacae Beutenmüller, 1897 & 3 \\
\hline Carmenta mariona (Beutenmüller, 1900) & 1 \\
\hline Carmenta mimuli (Edwards, 1881) & 39 \\
\hline Carmenta prosopis Edwards, 1882 & 3 \\
\hline Carmenta pyralidiformis Walker, 1856 & 17 \\
\hline Carmenta tecta Edwards, 1882 & 1 \\
\hline Carmenta texana Edwards, 1881 & 2 \\
\hline
\end{tabular}


Table 2. (continued) Species inventory of OSAC North American Sesiidae

\begin{tabular}{|c|c|}
\hline Taxon & \# specimen \\
\hline Carmenta verecunda Edwards, 1881 & 1 \\
\hline Euhagena emphytiformis (Walker, 1856) & 3 \\
\hline Euhagena nebraskae Edwards, 1881 & 11 \\
\hline Hymenoclea palmii Beutenmüller, 1902 & 4 \\
\hline Melittia calabaza Duckworth \& Eichlin, 1973 & 2 \\
\hline Melittia cucurbitae Harris, 1828 & 23 \\
\hline Melittia gloriosa Edwards, 1880 & 25 \\
\hline Melittia grandis Strecker, 1881 & 4 \\
\hline Melittia snowii Edwards, 1882 & 33 \\
\hline Palmia praecedens Edwards, 1883 & 1 \\
\hline Paranthrene asilipennis Boisduval, 1829 & 17 \\
\hline Paranthrene dollii Neumoegen, 1894 & 7 \\
\hline Paranthrene pellucida Greenfield \& Karandinos, 1979 & 4 \\
\hline Paranthrene robiniae (Edwards, 1880 ) & 51 \\
\hline Paranthrene simulans Grote, 1881 & 45 \\
\hline Paranthrene tabaniformis (Rottemburg, 1775) & 16 \\
\hline Pennisetia marginatum (Harris, 1839) & 8 \\
\hline Penstemonia clarkei Engelhardt, 1946 & 16 \\
\hline Penstemonia pappi Eichlin, 1987 & 6 \\
\hline Podosesia aureocincta Purrington \& Nielsen, 1977 & 13 \\
\hline Podosesia syringae Harris, 1839 & 90 \\
\hline Sannina uroceriformis Walker, 1856 & 5 \\
\hline Sesia apiformis (Clerck, 1759) & 3 \\
\hline Sesia spartani Eichlin \& Taft, 1988 & 3 \\
\hline Sesia tibiale (Harris, 1839) & 140 \\
\hline Sophona snellingi Eichlin, 1986 & 1 \\
\hline Synanthedon acerni (Clemens, 1860) & 15 \\
\hline Synanthedon acerrubri Engelhardt & 22 \\
\hline Synanthedon albicornis (Edwards, 1881) & 48 \\
\hline Synanthedon arizonensis Beutenmüller, 1916 & 1 \\
\hline Synanthedon bibionipennis Boisduval, 1869 & 277 \\
\hline Synanthedon castaneae Busck, 1913 & 4 \\
\hline Synanthedon chrysidipennis Boisduval, 1869 & 56 \\
\hline Synanthedon culiciformis (Linnaeus, 1758) & 5 \\
\hline Synanthedon decipiens Edwards, 1881 & 4 \\
\hline Synanthedon exitiosa Say, 1823 & 168 \\
\hline
\end{tabular}


Table 2. (continued) Species inventory of OSAC North American Sesiidae

Taxon
\begin{tabular}{|l|c|}
\hline Synanthedon fatifera Hodges, 1963 & 1 \\
\hline Synanthedon fulvipes (Harris, 1839) & 10 \\
\hline Synanthedon geliformis (Walker, 1856) & 1 \\
\hline Synanthedon helenis Druce, 1889 & 12 \\
\hline Synanthedon kathyae Duckworth \& Eichlin, 1977 & 1 \\
\hline Synanthedon mellinipennis Boisduval, 1836 & 20 \\
\hline Synanthedon novaroensis (Edwards, 1881) & 66 \\
\hline Synanthedon pictipes Grote \& Robinson, 1868 & 30 \\
\hline Synanthedon polygoni Edwards, 1881 & 16 \\
\hline Synanthedon proxima (Edwards, 1881) & 9 \\
\hline Synanthedon pyri (Harris, 1830) & 7 \\
\hline Synanthedon resplendens Edwards, 1881 & 232 \\
\hline Synanthedon rhododendri Beutenmüller, 1909 & 7 \\
\hline Synanthedon rileyana Edwards, 1881 & 17 \\
\hline Synanthedon sapygaeformis Walker, 1856 & 2 \\
\hline Synanthedon saxifragae Edwards, 1881 & 2 \\
\hline Synanthedon scitula (Harris, 1839) & 9 \\
\hline Synanthedon sequoiae (Edwards, 1881) & 125 \\
\hline Synanthedon sigmoidea (Beutenmüller, 1897) & 10 \\
\hline Synanthedon tipuliformis (Clerck, 1759) & 8 \\
\hline Vitacea admiranda (Edwards, 1882) & 1 \\
\hline Vitacea cupressi (Edwards, 1881) & 1 \\
\hline Vitacea polistiformis (Harris, 1854) & 29 \\
\hline Vitacea scepsiformis (Edwards, 1881) & 8 \\
\hline Zenodoxus heucherae Edwards, 1881 & 1 \\
\hline Zenodoxus palmii Neumoegen, 1891 & 6 \\
\hline Zenodoxus rubens Engelhardt, 1946 & \\
\hline Zenodoxus sidalceae Engelhardt, 1946 & 1 \\
\hline
\end{tabular}

Additional material from outside of North America is present, but was not digitized and catalogued at this time.

Label data for these specimens can be accessed via online data portals that serve osac IPT data (e.g., www.gbif.org) or by direct download via the archived datasets stored in the supplemental files of this paper. 


\section{Discussion:}

In the Pacific Northwest, Cossidae are most common and diverse east of the Cascade Mountains in association with their larval food plants that include aspen and willow in riparian habitats. However, one California species, Toronia perplexa, can be found uncommonly in coniferous forests along the west slope of the Cascade Range. Currently the OSAC's holdings of T. perplexa amount to but 5 specimens, 4 of which were taken at the H. J. Andrews Experimental Forest near Blue River, Oregon. More surveys of the western Cascades during their flight season could significantly expand the known range of this species in Oregon.

OSAC's holdings of North American Sesiidae are more comprehensive, including nearly $60 \%$ of the currently known North American fauna (Moths Photographers Group 2019; Hodges 1983). This is largely the result of material placed into the collection by Benjamin Garrison Thompson, who wrote his dissertation at Oregon State Agricultural College on clearwing moths (Thompson 1929) as well as Mr. John Vernon, an outstanding sesiid enthusiast who gave his collection to the university in 2008.

Minor corrections and other small modifications made in the future to this dataset will be accomplished by issuing a new version and providing a file of modified records to the supplemental files. Major additions, such as new records, new data fields, new contributors or other significant modifications will be accomplished through the generation and release of new datasets.

\section{Acknowledgments}

The records published herein were generated with funds from NSF: DBI\#1601888: Digitization TCN: Collaborative Research: Lepidoptera of North America Network: Documenting Diversity in the Largest Clade of Herbivores.

\section{References}

Barnes, W., J. H. McDunnough. 1911. Revision of the Cossidae of North America. Cont. Nat. Hist. Lepid. N. America. 1(i): [13-35, pls. I-VII, [1].

Hodges R.W. 1983. Check list of the Lepidoptera of America north of Mexico : including Greenland. E.W. Classey and Wedge Entomological Research Foundation; Distributors, The Foundation c/o National Museum of Natural History, London, Washington, D.C., xxiv, 284 pp.

Moths Photographers Group. 2019. Digital Guide to Moth Identification. Sesiidae. https:// mothphotographersgroup.msstate.edu/species list.php?plate $=06$ [accessed April 1, 2019].

Schoorl J.W. 1990. A phylogenetic study on Cossidae (Lepidoptera: Ditrysia) based on external adult morphology. Zoölogische Verhandelingen 263: 1-295.

Seltmann, K. C. et al. 2017. LepNet: The Lepidoptera of North America Network. Zootaxa 4247 (1): 073-077. https:/ / doi.org/10.11646/ zootaxa.4247.1.10.

Shepard J.H., C.J. Marshall, P.C. Hammond. 2018. North American Lepidoptera in the Oregon State Arthropod Collection: Hesperiidae, Pieridae, Papilionidae. Catalog: Oregon State Arthropod Collection 2(2): 1-2 and supplement file. doi: http://dx.doi.org/10.5399/osu/cat osac.2.2.4480

Thompson BG (1929) Moths of the family Aegeriidae occurring in Oregon, with notes. Pan Pacific Entomologist 5: 117-124. 